\title{
Atteintes Oculaires Au Cours Du Syndrome De Wolfram À Propos De Deux Cas Et Revue De La Littérature
}

\author{
Abba Kaka H.Y. \\ Service d'Ophtalmologie Hôpital National de Niamey, Niger \\ Guirou N. \\ Institut d'Ophtalmologie Tropicale de l'Afrique Bamako, Mali \\ Berete C.R.
}

Service d'Ophtalmologie Centre Hospitalo-universitaire de Treichville,

Cote d'Ivoire

Amza A.

Service d'Ophtalmologie Hôpital National Lamordé Niamey, Niger

\section{Daou $M$.}

Service de Médecine Interne Hôpital National de Niamey, Niger

doi: 10.19044/esj.2017.v13n27p269 URL:http://dx.doi.org/10.19044/esj.2017.v13n27p269

\begin{abstract}
Introduction: Wolfram syndrome is an autosomal recessive neurodegenerative disorder. Diabetes mellitus and juvenile bilateral optic atrophy are its major signs. It is recognized that this association, which started in childhood or during adolescence, is sufficient to diagnose Wolfram syndrome. Optic atrophy occurs in $98 \%$ to $100 \%$ of cases with an average age of onset of 11 years. We reported a study of two confirmed cases referred by the internal medicine department. Observations: Case 1: A 23year-old woman, deaf and dumb by birth, went through a diabetic ketosis test. Ophthalmologic examination showed reduced visual acuity in the fingers at 5 meters P2 in both eyes. Also, the fundus of the eye showed bilateral atrophic papillary palpation with no signs of retinopathy. She had deafness of deep perception and hypogonadotropic hypogonadism. Deafness, diabetes, optic atrophy, and hypogonadism led to the diagnosis. Case 2: A 21-year-old man born from a first-degree consanguineous marriage serves as a supplement to the management of diabetes. The visual acuity was at counting fingers at $1 \mathrm{~m}$ to the right eye and sees the hand move to $0.5 \mathrm{~m}$ to the left eye. On examination at the slit lamp, it had a bilateral dense cataract. After phacoexeresis, the base revealed bilateral optic atrophy. Ultrasound of the urinary tree showed hypotonia of the renal cavities and a neurogenic
\end{abstract}


bladder. Also, audiometry showed mild sensory deafness. The diagnosis of Wolfram syndrome was made in front of the tetrad: diabetes, optic atrophy, deafness, and urinary signs. Discussion: Wolfram syndrome may be familial or sporadic. The gene however is located on the short arm of chromosome 4. Optic atrophy is secondary to the involvement of pre-genetic fibers, and it is characterized initially by temporal palpation of the papilla. The evolution towards diffuse whitish discoloration occurs in a few months or years with the gradual establishment of a blindness around the age of 17 to 30 years. Conclusion: Wolfram syndrome is a clinical entity characterized by clinical and genetic polymorphism. This diagnosis should be considered in the presence of any type I diabetes associated with optic atrophy in children.

Keywords: Wolfram syndrom, diabetes mellitus, deafness, optic atrophy, Niger

\section{Résumé}

Introduction: Le syndrome de Wolfram est une affection neurodégénérative autosomique récessive. Le diabète sucré et l'atrophie optique bilatérale juvéniles en sont ses manifestations majeures, il est admis que cette association ayant débuté dans l'enfance ou durant l'adolescence suffit pour poser le diagnostic du syndrome de Wolfram. L'atrophie optique est présente dans $98 \%$ à $100 \%$ des cas avec un âge moyen d'apparition de11 ans. Nous rapportons une étude sur deux cas confirmés référés par le service de médecine interne. Observations: Cas no 1: Il s'agissait d'une femme âgée de 23 ans, sourde et muette de naissance, vue dans le cadre d'un bilan de cétose diabétique. L'examen ophtalmologique a objectivé une acuité visuelle réduite à compte les doigts à 5 mètres $\mathrm{P} 2$ aux deux yeux, et le fond d'œil a montré une pâleur papillaire atrophique bilatérale sans signes de rétinopathie. Elle avait une surdité de perception profonde et un hypogonadisme hypogonadotrope. L'association surdité, diabète, atrophie optique et hypogonadisme a permis de poser le diagnostic. Cas no 2 : Il s'agissait d'un patient âgé de 21 ans, issu d'un mariage consanguin au premier degré, vu pour complément de prise en charge d'un diabète. L'acuité visuelle était à compte les doigts à $1 \mathrm{~m}$ à l'œil droit et voit bouger la main à $0,5 \mathrm{~m}$ à l'œil gauche. A l'examen à la lampe à fente il avait une cataracte dense bilatérale. Après phacoexérèse, le fond'œil révéla une atrophie optique bilatérale. L'échographie de l'arbre urinaire a montré une hypotonie des cavités rénales et une vessie neurogène, l'audiométrie a montré une surdité neurosensorielle légère. Le diagnostic du syndrome de Wolfram a été posé devant la tétrade : diabète, atrophie optique, surdité et les signes urinaires. Discussion : Le syndrome de Wolfram peut être familial ou sporadique, le gène étant situé sur le bras court du chromosome 4. L'atrophie optique est secondaire à une atteinte des fibres optiques prégéniculées, et se caractérise 
au début par une pâleur papillaire localisée en temporal. L’évolution vers la décoloration blanchâtre diffuse se fait en quelques mois ou années avec l'installation progressive d'une cécité vers l'âge de 17 à 30 ans. Conclusion: Le syndrome de Wolfram est une entité clinique caractérisée par un polymorphisme clinique et génétique. Ce diagnostic doit être envisagé devant tout diabète de type I associé à une atrophie optique chez l'enfant.

Mots-clés: Syndrome de Wolfram, atrophie optique, diabète sucré, Niger

\section{Introduction}

Le syndrome de Wolfram, ou DIDMOAD (pour Diabetes Insipidus, Diabetes Mellitus, Optic Atrophy and Deafness), est une maladie génétique rare de transmission autosomique récessive. Elle est responsable de la constitution pendant les trois premières décennies de la vie d'un tableau complexe associant diabète juvénile, atrophie optique, diabète insipide central, surdité, manifestations neurologiques centrales, périphériques et végétatives. Le gène responsable a été localisé en $4 \mathrm{p} 16.1$ pour le type 1 et au niveau du locus 4q22-24 pour le type 2(Vialettes et al., 2013). L'atrophie optique est secondaire à une atteinte des fibres optiques prégéniculées, est souvent bilatérale et symétrique et se caractérise au début par une pâleur papillaire localisée en temporal. L'évolution vers la décoloration blanchâtre diffuse se fait en quelques mois ou années avec l'installation progressive d'une cécité vers l'âge de 17 à 30 ans (Skiker et al., 2007). Nous rapportons l'observation de deux patients présentant un syndrome de Wolfram avec des circonstances de découverte et des signes cliniques différents dans chacun des cas. À travers ces deux cas et une revue de la littérature les auteurs exposent les complications ophtalmologiques de ce syndrome ainsi que ses aspects cliniques.

\section{Patients et Méthodes \\ Observations}

\section{Cas $N^{\circ} 1$}

A.Z. âgée de 23 ans, sourde et muette de naissance, issue d'un mariage non consanguin, nous a été adressée pour complément de prise en charge d'une cétose diabétique inaugurale. L'interrogatoire ressort que les premiers symptômes du diabète étaient depuis l'âge de 9ans sans notion de prise en charge particulière. Elle se plaignait d'une baisse d'acuité visuelle progressive depuis l'âge de 10 ans. L'examen ophtalmologique a objectivé une acuité visuelle à compte les doigts à 5 mètres P2 aux deux yeux, l'examen du fond d'œil a montré une pâleur papillaire atrophique bilatérale sans signes de rétinopathie (Figure 1). L'audiométrie a révélé une surdité de perception profonde avec un seuil auditif à $103 \mathrm{~dB}$ à l'oreille droite et 
$93.3 \mathrm{~dB}$ à l'oreille gauche. Le bilan hormonal a objectivé un hypogonadisme hypogonadotrope FSH : $7.48 \mathrm{U} / \mathrm{l}(3-8 \mathrm{U} / \mathrm{l}) ; \mathrm{LH}: 4.88 \mathrm{U} / 1(2.5-21 \mathrm{U} / \mathrm{l})$ Estradiol : 26.25pg/ml (94-508 pg/ml) ; Progestérone : $0.14 \mathrm{ng} / \mathrm{ml}(3,2-38,6$ $\mathrm{ng} / \mathrm{ml}$ ). L'échographie de l'arbre urinaire était normale L'association surdité, diabète, atrophie optique et hypogonadisme a permis de conclure le diagnostic du syndrome de Wolfram chez cette patiente.

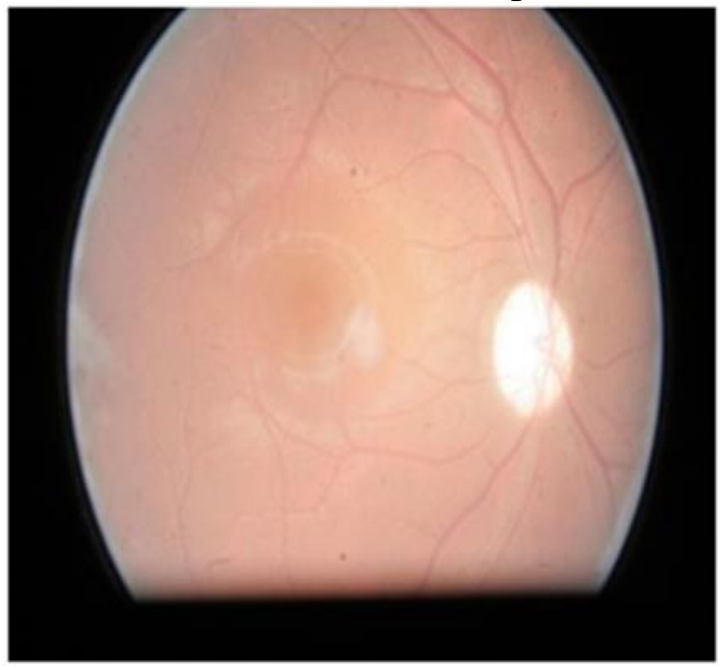

Figure 1. Pâleur papillaire atrophique

Tableau 1. Signes diagnostics du syndrome de wolfram chez la patiente no 1

\begin{tabular}{|c|c|c|c|}
\hline $\begin{array}{c}\text { Eléments } \\
\text { diagnostiques }\end{array}$ & Critères majeurs & $\begin{array}{c}\text { Critères } \\
\text { mineurs }\end{array}$ & Autres signes évocateurs \\
\hline Système endocrinien & Diabète Type I & & $\begin{array}{c}\text { - Hypogonadisme } \\
\text {-Absence d'immunité } \\
\text { anti-cellule } \beta \\
\text { pancréatique }\end{array}$ \\
\hline Atteintes sensorielles & Atrophie optique & $\begin{array}{c}\text { Surdité } \\
\text { neurosensorielle }\end{array}$ & \\
\hline
\end{tabular}

\section{Observation2}

A.B. âgé de 21 ans, issu d'un mariage consanguin au premier degré, nous a été adressé pour complément de prise en charge d'un diabète inaugural. L'interrogatoire révèle que le début de la symptomatologie remonterait à 4 ans par un syndrome polyuro-polydypsique. L'évolution est marquée par l'installation progressive d'un amaigrissement et d'une baisse progressive de la vision depuis 2 ans associée à des paresthésies à type de chaleur aux 2 membres inférieurs et de sensation de vessie pleine. L'acuité visuelle était à compte les doigts à $1 \mathrm{~m}$ à 1 'œil droit et réduite à une perception lumineuse à l'œil gauche. A l'examen à la lampe à fente il avait une cataracte cortico-sous capsulaire dense bilatérale plus à gauche. Après 
phacoexérèse et la pose d'un implant de chambre postérieure, l'acuité était de 1/10 P2 aux deux yeux avec la meilleure correction. Le fond'œil révéla une pâleur papillaire atrophie bilatérale (Figure 2). Le champ visuel a montré un rétrécissement concentrique et la vision des couleurs a ressorti une altération sur l'axe rouge vert. Le bilan général a montré une altération profonde de l'état général avec un IMC $=13.43 \mathrm{Kg} / \mathrm{m}^{2}$. Le bilan hormonal a montré un hypogonadisme hypogonadotrope avec un taux de testostérone bas à $2.49 \mathrm{ng} / \mathrm{ml}$ (16-30mmol/l). L'échographie de l'arbre urinaire a objectivé une hypotonie des cavités rénales droite et gauche avec une vessie en pleine réplétion contenant $550 \mathrm{ml}$. Le résidu post mictionnel était très significatif à $318 \mathrm{ml}$ (vessie neurogène). L'audiométrie (Figure 3) a montré une surdité neurosensorielle légère (seuil auditif à $16.66 \mathrm{~dB}$ à l'oreille droite et $13.33 \mathrm{~dB}$ à l'oreille gauche). Le diagnostic du syndrome de Wolfram a été posé devant la tétrade : diabète, atrophie optique, surdité et les signes urinaires.

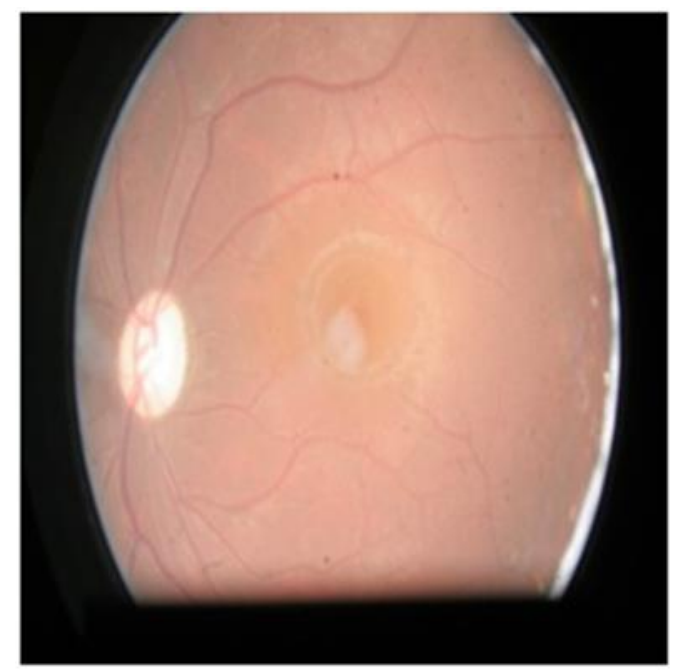

Figure 2. Pâleur papillaire atrophique

Tableau 2. Signes diagnostics du syndrome de wolfram chez le patient no 2

\begin{tabular}{|c|c|c|c|}
\hline $\begin{array}{c}\text { Eléments } \\
\text { diagnostiques }\end{array}$ & Critères majeurs & Critères mineurs & $\begin{array}{c}\text { Autres signes } \\
\text { évocateurs }\end{array}$ \\
\hline Système endocrine & Diabète Type I & $\begin{array}{c}\text { - Hypogonadisme } \\
\text {-Absence d'immunité } \\
\text { anti-cellule } \beta \\
\text { pancréatique }\end{array}$ \\
\hline Atteintes sensorielles & Atrophie optique & $\begin{array}{c}\text { Surdité } \\
\text { neurosensorielle }\end{array}$ & Cataracte \\
\hline Génétique & & & \\
\hline
\end{tabular}




\begin{tabular}{|c|c|}
\hline Autres & $\begin{array}{c}\text {-Signes } \\
\text { neurologiques } \\
\text {-Troubles } \\
\text { urologiques }\end{array}$ \\
\hline
\end{tabular}

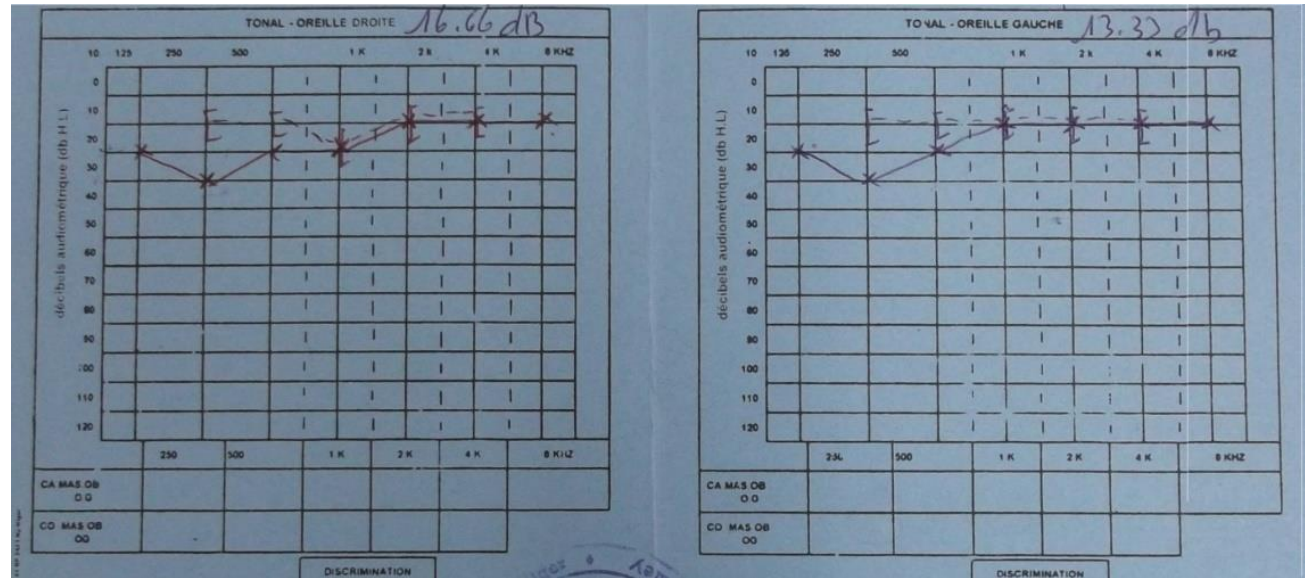

Figure 3. Audiometrie montrant une surdité neurosensorielle légère

\section{Discussion}

La première description du syndrome de Wolfram a été faite en 1859 par Von Graefe qui rapporte l'association d'un diabète sucré et d'une atrophie optique chez un jeune garçon. En 1938 Wagner et Wolfram ont décrit cette même association chez quatre enfants d'une même fratrie de huit (Lombardo et al., 2005). Actuellement les auteurs s'accordent à redonner son importance à la classique tétrade du DIDMOAD (diabetes insipidus, diabetes mellitus, optic atrophy, deafness) retrouvée dans 10 à $53 \%$ des cas (Lombardo et al., 2005). Les deux signes majeurs sont le diabète sucré et l'atrophie optique bilatérale. Il est admis que cette association (diabète sucré et atrophie optique bilatérale) ayant débuté dans l'enfance ou durant l'adolescence suffit pour poser le diagnostic du syndrome de Wolfram (Benchekroun et al., 2001). D'un point de vue chronologique, le diabète sucré apparaît durant la première décade de la vie, suivi de l'atrophie optique. Cette séquence est la plus commune mais n'est toutefois pas obligatoire, l'atrophie optique pouvant être inaugurale. L'âge moyen d'apparition du diabète est 6 ans (avec des extrêmes variant entre 3 semaines de vie et 16 ans) et celui de l'atteinte oculaire 11 ans (avec des extrêmes comprises entre 6 semaines d'âge et 19 ans). Au cours de la deuxième décade se développent le diabète insipide et la surdité de perception. L'atteinte urologique est constatée le plus souvent au début de la troisième décennie. Le syndrome de Wolfram est une affection rare (Sayouti et al., 2007). Au cours de l'étude de 
Barrett $\mathrm{T}$ et al la prévalence de ce syndrome a été estimée à environ 1/770000 habitants en Angleterre et à 1/100000 habitants en Amérique du Nord (Barrett et al., 1995). La prévalence de ce syndrome dans la population infantile (enfant ayant moins de 15 ans d'âge) avait été estimée par Fraser et Gunn à 1/100000 en se basant sur la fréquence de survenue de l'atrophie optique dans la population diabétique juvénile, qui était 1/148. En 1992, Barrett $T$ va évaluer cette prévalence infantile à $1 / 500000$, en se basant sur le nombre de sujets ayant le syndrome de wolfram dans la population totale en Angleterre (Barrett et al., 1995; Fraser et al., 1977). Le syndrome de Wolfram touche indifféremment les deux sexes, comme le montre l'étude de Barrett $\mathrm{T}$, en 1995, qui comprenait 45 patients atteints du syndrome de wolfram, soit 24 femmes et 21 hommes. L'étude de Kinsley et al. faite chez 68 patients atteints de syndrome de wolfram, comprenait 35 hommes et 33 femmes provenant de 44 familles, recensées dans 23 états des Etats-Unis d'Amérique (Kinsley, 1995).Ce syndrome peut être familial ou sporadique. Pour les cas familiaux la transmission se fait sur un mode autosomique récessif, le gène du Wolfram étant situé sur le bras court du chromosome 4. Il a récemment été identifié un gène dénommé "Wolframin" codant pour une protéine membranaire de différents tissus, incluant le cerveau et le pancréas notamment; ce gène verrait une perte de fonction due à une mutation au niveau de ses deux allèles chez les patients atteints de syndrome de Wolfram (Smith et al., 2004). L'IRM révèle une atrophie généralisée du cerveau, particulièrement du cervelet, du bulbe rachidien et du pont, une absence de signal du lobe pituitaire postérieur et un signal réduit du nerf optique. Le diagnostic est confirmé par l'analyse génétique. Le diagnostic différentiel inclut les maladies mitochondriales tels les diabètes transmis par la mère et la surdité, la neuropathie optique héréditaire de Leber, l'anémie mégaloblastique thiamine-dépendante, l'atrophie optique autosomique dominante plus et le syndrome de Mohr-Tranebjaerg (Pakdemirli et al., 2005).

Les manifestations ophtalmologiques sont dominées par l'atrophie optique retrouvée dans 98 à 100\% des cas. L'atteinte oculaire est due à une atrophie progressive de tout le système visuel à savoir nerf optique, chiasma et bandelettes optiques, dont l'origine est mal définie. Elle est indépendante du diabète sucré. Elle entre dans le cadre d'une atteinte nerveuse centrale probable (Medlej et al., 2004). L'atteinte oculaire est retrouvée chez 35 des 45 patients de l'étude de Barrett $\mathrm{T}$ et al soit $78 \%$ des cas et chez $100 \%$ des cas de l'étude de R.Medlej et al. Elle entraîne une dégradation progressive de l'acuité visuelle amenant le patient à consulter, ou elle peut être découverte lors d'un examen systématique chez un enfant diabétique, d'où l'intérêt de la connaissance de cette affection afin de guider le patient vers les explorations nécessaires (Fraser et al., 1977). Sur le plan clinique, elle se caractérise par 
une perte de vision de couleurs, rapidement suivie d'une diminution de l'acuité visuelle, qui aboutit en 5 à 10 ans à la cécité. Le fond d'œil finira par poser le diagnostic d'atrophie optique bilatérale à un stade avancé de l'évolution. C'est le cas pour nos deux patients qui ont été diagnostiqué à un stade tardif avec atrophie optique bilatérale. Les anomalies pigmentaires de la rétine périphérique sont rares dans ce syndrome (Meyer et al., 2004). L'étude de champ visuel à un stade précoce de la maladie, va alors montrer un scotome central ou une constriction de champ visuel périphérique. Les potentiels évoqués visuels mettent en évidence des anomalies, qui concordent avec une atteinte du nerf optique chez tous les patients, l'électrorétinogramme est normal dans la majorité des cas. L'imagerie par résonance magnétique, pratiquée précocement, va mettre en évidence un hyposignal et une atrophie bilatérale des nerfs optiques, du chiasma et des bandelettes optiques (Mandeep et al., 1994). D'autres atteintes ophtalmologiques ont été décrites : atteinte de la musculature extrinsèque à type de strabisme et ptosis, nystagmus, atteinte de la musculature intrinsèque avec réflexe photomoteur paresseux puis absent au stade d'atrophie optique constituée, anomalies du segment antérieur à type d'embryotoxon postérieur (Mandeep et al., 1994; Garcia-Luna et al., 1988; Bernard et al., 1987). La rétinopathie diabétique est liée à la durée du diabète, sa survenue dans le cadre du syndrome de Wolfram est rare et estimée à $7 \%$. La cause de ce faible taux de rétinopathie chez ces patients n'est pas encore connue, malgré la survenue précoce du diabète et la difficulté de sa prise en charge (Mandeep et al., 2006; Seynaeve et al., 1994). Bernard et al ont retrouvé 15 cas de rétinopathie diabétique sur 200 patients atteints de Wolfram (Bernard et al., 1987). Barrett et al eux ont retrouvé 3 cas sur 45 patients (Barrett et al., 1995). Nos deux patients n'avaient aucun signe de rétinopathie diabétique malgré plus de 10 ans de diabète. Dans la littérature, il y a très peu de données sur les opacités cristalliniennes associées au syndrome de wolfram. Les rares allusions n'ont donné aucune spécificité sur le type de cataracte (Cremers et al., 1977). Certains auteurs ont décrit la présence d'une cataracte corticale postérieure, ou d'une cataracte ponctuée, cette dernière a été décrite par Francisco et al chez un enfant âgé de 13 ans (Francisco et al., 2000). Notre second patient a présenté une cataracte cortico-sous capsulaire postérieure dense bilatérale.

\section{Conclusion}

Le syndrome de wolfram est une maladie grave de pronostic sombre avec une espérance de vie qui ne dépasse pas la trentaine. Chez tout enfant diabétique, il faut surveiller le fond'œil, le champ visuel et la vision des couleurs, à la moindre anomalie de ces examens complémentaires, on doit suspecter la maladie de wolfram et indiquer systématiquement une IRM afin 
de faire un diagnostic précoce et une prise en charge efficace et ainsi prolonger l'espérance de vie des malades et sûrement d'améliorer la qualité de cette survie.

\section{References:}

1. Barrett T.G, Bundey S.E, Maclead A.F., \& coll (1995). Neurodegeneration and diabetes: UK nationwide study of wolfram (DIDMOAD) syndrome; The lancet, 12, 346, 1458-1462.

2. Benchekroun, A., Nouini, Y., Iken, A., Iraqui, M., \& Coll (2001). Le syndrome de Wolfram. A propos de 3 cas ; Progrès en Urologie; 11, 1292-1297.

3. Bernard, C. \& Grange, JD. (1987). Le syndrome de Wolfram ou DIDMOAD syndrome. Bull Soc Opht France, 5: 651-653.

4. Cremers, CWRJ., Wijdeveld, PGAB., \& Pinckers, AJLG. (1977). Juvenile diabetes mellitus, op- tic atrophy, hearing loss, diabetes insipidus, atonia of the urinary tract and bladder, and other abnormalities (Wolfram syndrome); Acta Paediatr Scand, 264, 1-16.

5. Francisco, J., Castro, J., Babrio, M., \& Francisca, P. (2000). Uncommon ophthalmological findings associated with Wolfram syndrome, Acta Ophthalmol Scand, 78: 118- 119.

6. Fraser, FC. \& Gunn, T. (1977). Diabetes mellitus, diabetes insipidus, and optic atrophy. An autosomal recessive syndrome? J.Med.Genet, 14, 190-3.

7. Garcia-Luna, PP., Villechenous, E., Leal-Cerro, A., Duran, S., Jorge, S., Wichmann, I., Nunez- Roldan, A., \& Astorga, R. (1988). Contrasting features of insulin dependent diabetes mellitus associated with neuroectodermal defects and classical insulin dependent diabetes mellitus. Acta Paediatr Scand, 77, 413-418.

8. Kinsley, B.T., Swift, M., Dumont, R.H., \& Swift, R.G. (1995). Morbidity and mortality in the Wolfram syndrome. Diabetes Care, December, 12, 18, 12, 1566-1570.

9. Lombardo, F., Chiurazzi, P., \& Hortnagel, K. (2005). Clinical picture, evolution and peculiar mo- lecular findings in a very large pedigree with Wolfram syndrome. J Pediatr Endocrinol Me- tab, 18:1391-7.

10. Mandeep, S.D., Uday, R.D., \& Zucker- Brod, D.S. (2006). Pigmentary maculopathy in a patient Mandeep S.D with Wolfram syndrome. Can J Ophthalmol, 41:38-40.

11. Medlej, R., Wasson, J., Baz, P., Azar, S., Salti, I., Loisel, J., \& coll (2004). Diabetes Mellitus and Optic Atrophy: A study of Wolfram Syndrome in the Lebanese population The Journal of Clinical 
endocrinology and Metabolism The Endocrine-society, 89, 4, 16561661.

12. Meyer, C.H., Rodrigues, E.B., \& Schmidt, J.C. (2004). Bilateral progressive optic atrophy and without diabetic retinopathy in a young diabetic patient. Wolfram syndrome: diabetes insipidus, diabetes mellitus, optic atrophy and deafness (DIDMOAD). Ophthalmologe, 101:394-6.

13. Pakdemirli, E., Karabulut, N., Bir, LS., \& Sermezy (2005). Cranial magnetic resonance imaging of Wolfram syndrome (DIDMOAD) Radiologie Australienne, 4, 2, 49, 189.

14. Sayouti \& coll (2007). Syndrome de wolfram à propos de deux cas, $\mathrm{j}$ fr. ophtalmol, 2007, 30, 6, 607-609.

15. Seynaeve, H., Vermeiren, A., Leys, A., \& Lands, L. (1994). Four cases of Wolfram syndrome: ophthalmologic findings and complications. Bull Soc belge Ophtalmol. 252:75-80.

16. Skiker, H., Boutimzine, N., Ouazani, B., Kerman, A., Laghmari, M., Ibrahimy, W., \& Daoudi, R. (2007). Le Syndrome De Wolfram, à propos de quatre cas ; Bull. Soc. belge Ophtalmol., 306, 43-47.

17. Smith, C.J., Crockpa, King, B.R., Meldrum, C.J., \& Scott, R.J. (2003). Phenotype-genotype correlations in a series of Wolfram syndrome families, Diabetes Care, 27: -9.

18. Vialettes, B. \& Dubois-Léonardon, N. (2013). Le syndrome de Wolfram : une maladie "orpheline » que les diabétologues ne peuvent pas ignorer, Médecine Des Maladies Métaboliques, 12, 7, 6 , 513-519. 